\title{
Research on Event-driven Data Collection Protocol of Wireless Sensor Networks
}

\author{
https://doi.org/10.3991/ijoe.v12i10.6205 \\ Cunjiang $\mathrm{Yu}$ \\ Changchun University, Changchun, China
}

\begin{abstract}
This paper carries out research on two types of event-driven data collection protocols of wireless sensor networks: EDDGP and ACBP protocols. In the EDDGP protocol, an adaptive evaluation model is put forward to select the cluster head, including the node classification, the fuzzy grade classification, the evaluation function and the parent grid resolution. Once an event of interest occurs, the member nodes will send the collected information to the cluster heads which will then integrate the data and send a request packet for data collection to the sink. After receiving the request packet, the sink will quickly move to the cluster head for collecting information. If the sink receives the request packet for data collection from other cluster heads of information source in the moving process, it will save their grid ID. The data from the other cluster heads of information source are collected in turn from the nearest ones to the farthest ones. The theoretical research and simulation results show that EDDGP can reduce the energy consumption of location updates of the sink so as to balance the energy consumption of nodes and prolong the lifetime of the network.
\end{abstract}

Index Terms-Data collection, wireless sensor networks, event-driven.

\section{INTRODUCTION}

The protocol of wireless sensor network data can be divided into two types which are active reporting and event-driven, according to different data transmission modes. In terms of the data collection protocol of the active reporting type, it will send the collected information to the sink actively when the nodes monitor the event of interest, such as the EBRP protocol in the previous paper. In terms of the event-driven data collection protocol, after the event of interest occurs, the sink will move to its vicinity for data collection. In this paper, the event-driven type of data collection protocol is studied[1].

\section{EVENT-DRIVEN DATA COLLECTION PROTOCOL OF WIRELESS SENSOR NETWORKS}

In order to prolong the lifetime of the network, in this section, an event-driven data collection protocol of wireless sensor network is put forward. This protocol proposes an adaptive evaluation model to select cluster heads which includes several steps as node classification, fuzzy classification, evaluation function and parent grid resolution. When the sink receives the data request packet, it moves to the cluster heads of the information source and starts to collect the event of interest within the cluster[2].

\section{A. Hypothetical condition}

It takes the hypothesis into consideration that one mobile sink and $\mathrm{N}$ sensor nodes collect data in the network $(L \times L)$ (seen in Figure 1).

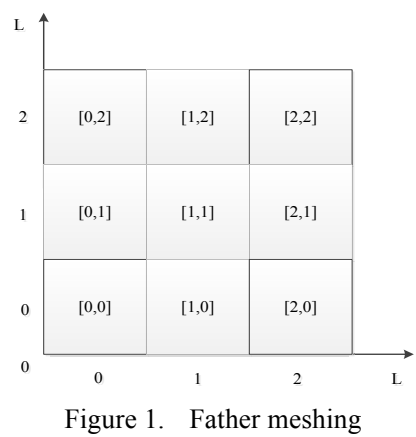

\section{B. EDDGP protocol}

Cluster heads of information source refers to some cluster head which sends the request packet of data collection to the sink after receiving the event of interest occurring within the cluster[3].

In the parent grid, a candidate region for cluster head is set up, which is shown in Figure 2. Cluster head candidate region is within the dotted line $\left(l_{i} \times l_{i}\right)$. In the region:

$$
l_{i}=2 l / w \quad(2 \leq w \leq 9)
$$

The nodes will judge whether or not they are candidate nodes based on the equation (2):

$$
\left\{\begin{array}{l}
2 l N_{x}^{i}+\frac{2 l-l_{i}}{2} \leq x \leq 2 l N_{x}^{i}+\frac{2 l-l_{i}}{2} \\
2 l N_{y}^{i}+\frac{2 l-l_{i}}{2} \leq x \leq 2 l N_{y}^{i}+\frac{2 l-l_{i}}{2}
\end{array}\right.
$$

In order to balance the energy consumption of the network, the fuzzy theory is adopted to divide the residual energy of the candidate nodes in the parent grid into a higher grade and a lower grade[4].

Equation (3) in the evaluation function:

$$
f=\alpha f_{D}+\beta f_{C}
$$

The formula is an evaluation function which will judge the importance of a node as cluster head from the two aspects of the number of neighbors and the distance to the center of the parent grid. Function (3) is $f_{n}=h_{i} / h_{\text {neighbor }}$, $f_{c}=\left(D_{c}-D_{t}\right) / D_{c}$ and $h_{\text {neighbor }}$ represents the threshold of neighbor nodes; $h_{i}$ represents the number of adjacent nodes for candidate node $\mathrm{i} ; f_{n}$ can judge the importance of the node as cluster head from the perspective of the 
number of adjacent nodes it has; $D_{c}$ represents the distance from the vertex to the center point in the candidate region[5]; $D_{i}$ represents the distance of the candidate node $i$ from the center point; $f_{c}$ can judge the importance of a node as the cluster head from the aspect of its distance from the center of parent grid. The nodes with relatively more neighboring nodes and which are closer to the center point will be selected as the cluster heads[6]. $\alpha, \beta$ are the regulation factors. The node is able to calculate the coordinates $\left(x_{\text {center }}, y_{\text {center }}\right)$ of the grid center point according to the formula (4), the range of $\left(x_{\text {center }}, y_{\text {center }}\right)$ is shown in Figure 3.

$$
\left\{\begin{array}{l}
x_{\text {center }}=\left(2 N_{x}^{i}+1\right) l \\
y_{\text {center }}=\left(2 N_{y}^{i}+1\right) l
\end{array}\right.
$$

In the adaptive evaluation model, the number of candidate nodes can be adjusted by the change of the $\omega$ value[7]. When the parent grid has become the cluster for the first time and if the $\omega$ value is too small, the number of candidate nodes will be more than if the $\omega$ value is large, so as to increase the number of comparisons of nodes in terms of the $f$ value[8]. In this regard, the $\omega$ value is linked with the residual energy of the candidate nodes. The specific setup of $\omega$ value is shown in Table 1:

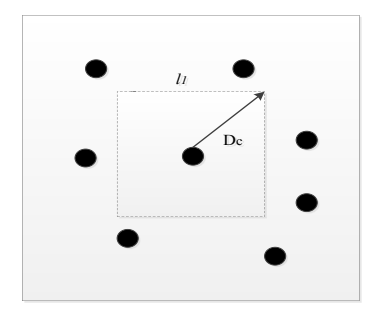

Figure 2. Cluster heads candidate regions

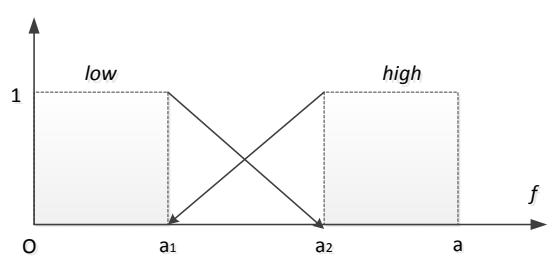

Figure 3. Fuzzy hierarchy

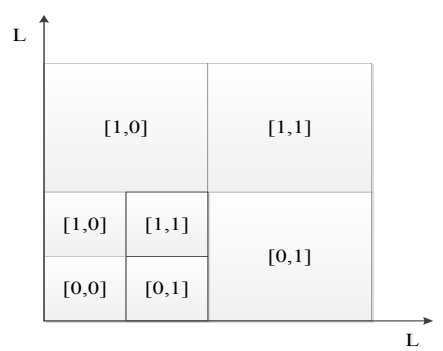

Figure 4. Sub-grid

In order to balance the energy consumption of nodes, when the average residual energy of the candidate nodes in the parent grid is less than $50 \%$, the parent grid will be split into four sub-grids of equal areas, which is shown in Figure 4 . The parent grid $[0,0]$ is divided into four subgrids: $[0,0],[0,1],[1,0],[1,1]$. The nodes in the sub-grids will be calculated to judge which one they belong to according to the equation (5) and then the cluster heads are selected according to the fuzzy theory and the evaluation function[9].

$$
\left\{\begin{array}{l}
2 l N_{x}^{i}+\frac{2 l-l_{i}}{2} \leq x \leq 2 l N_{x}^{i}+\frac{2 l-l_{i}}{2} \\
2 l N_{y}^{i}+\frac{2 l-l_{i}}{2} \leq x \leq 2 l N_{y}^{i}+\frac{2 l-l_{i}}{2}
\end{array}\right.
$$

The candidate nodes will report the residual energy to the cluster head with $h_{i}$ and $D_{i}$ attached in the message. At the end of each round, the cluster head calculates the average residual energy of the candidate nodes. Furthermore, the cluster head for the next round is selected according to the equations (1) and (2), the fuzzy theory and the evaluation function[10].

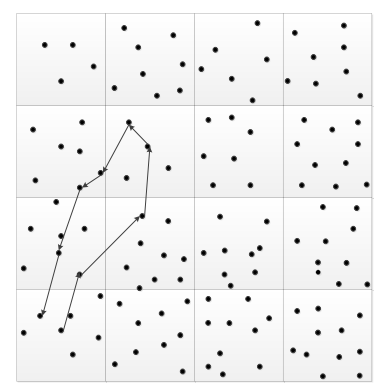

Figure 5. Sink movement diagram

The adaptive evaluation model has comprehensively taken the effect of residual energy of a node, the distance to the grid center and the number of neighboring nodes on cluster heads into consideration and adopts the fuzzy theory, changing value, evaluation function and parent grid resolution to adjust the selection of cluster heads[1113].

(3) The sink moving strategy based on event-driven type of protocol

After receiving a request packet for data collection sent by the cluster head of information source, the sink will move to its one-hop range for collecting information within the cluster. Before moving, the sink will broadcast the grid ID of the cluster head of information source, which is shown in Figure 5[14]. The black square is the cluster head while the triangle is the sink. If the sink receives the data collection request packets sent by another cluster head of information source in the process of moving, it will save their network ID[15]. After collecting the data of the current cluster heads of information source, the data of other cluster heads of information source are collected in turn from the nearest ones to the farthest ones[16].

\section{Performance analysis}

In EDDGP, after receiving a request package of an event of interest, the sink will move to the surrounding area of the cluster head of information source for collecting data so as to avoid the energy consumption brought about by relay forwarding in a data multiple hop; while in ALURP, each node will send the events of interest perceived by the sink through the relay forwarding in multiple hop. Obviously, EDDGP consumes less energy in the process of data collection. Next, this paper will compare the amount of energy consumption brought 
about by a location update of the sink in the two protocols[17].

It is assumed that within the time $\mathrm{T}$, the sink will move from the adaptive target area to the new grid for $m$ times $(\mathrm{m}>1)$. According to the ALURP protocol, the energy consumption caused by the location update of the sink is:

$$
E_{\text {ALURP }}=L^{2} \rho e+L^{2} \rho m
$$

where, $L^{2} p e$ is the energy consumed by all nodes to update the sink position when the network is initialized; $L^{2} p e$ is the energy consumption for the node to update the sink position when the sink moves out of the adaptive area for $\mathrm{m}$ times[18].

Likewise, it is inferred that the energy consumption of the cluster head of EDDGP is $L^{2} p e$. The forwarding region within the parent grid is shown in the shadow area of Figure.6 (a). When the parent grid is split into sub-grids, the forwarding area is shown in the shadow area of Figure 6 (b) with its area being $4\left(2 \sqrt{2} l d-2 d^{2}\right)$ and $r 0 \leq d \leq \sqrt{2} l / 2 . r_{0}$ is the communication radius of nodes. When all the parent grids are split into sub-grids, the energy consumption caused by the location update of the sink is:

$$
E_{E D D G P}=L^{2} \rho e+\frac{L^{2}}{l^{2}}\left(2 \sqrt{2} l d-2 d^{2}\right) \rho e+\frac{L^{2}}{l^{2}}\left(2 \sqrt{2} l d-2 d^{2}\right) \rho e m\left(r 0 \leq d \leq \frac{\sqrt{2}}{2} l\right)
$$

It can be inferred that in the EDDGP protocol, when the node communication radius is $r 0<((\sqrt{2}-1) / 2) l$, the energy consumption caused by the location update of the mobile sink is less than that in the ALURP protocol[19].

\section{Simulation experiment}

The setup of the experimental parameters are shown in the following table: According to the conversion, each side length (2L) of the grid in the EDDGP protocol corresponded to the optimal adaptive region in the ALURP protocol of $180 \mathrm{~m}$.

(1) The influence of node density

This section investigates the influence of node density on the network energy consumption with the node density being $0.001-0.005 / \mathrm{m}^{2}$ and the other parameters being the default values[20]. Figure 7 shows the effect of node density on the primary energy consumption of the sink position update for one time in the ALURP protocol. When the node density is equal, the energy consumption of the sink position update in the ALURP protocol is higher than that in the EDDGP protocol. When the node density increases, the energy consumption in ALURP increases sharply while the energy consumption in EDDGP increases slowly[21]. This is because in EDDGP when the node density is smaller, the candidate nodes are fewer, which cannot fully reflect the advantages of the evaluation model of adaptive cluster heads. Therefore, the difference in energy consumption in EDDGP and ALURP is less. When the node density increases, the candidate nodes are increased and the evaluation model of adaptive cluster heads can be used to select the optimal node as the cluster head. Consequently, the increasing intensity of energy consumption in EDDGP is much smaller than that in ALURP protocol. When the node density is $0.003 / \mathrm{m} 2$, the energy consumption of the sink position update in EDDGP is about $0.015 \mathrm{~J}$ while the energy consumption in ALURP protocol is about $0.05 \mathrm{~J}$, shown in Table 2.
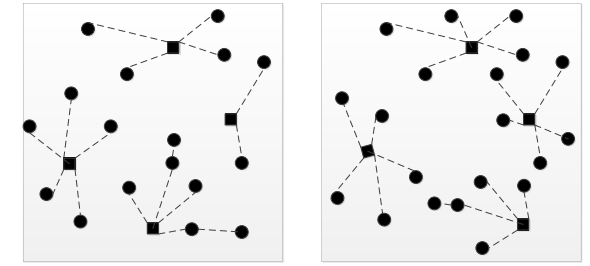

Figure 6. The influence of the cluster head position

(2) The effect of regulatory factors A and P

This section investigates the effect of ratio of regulating factors $\alpha, \beta$ on energy consumption. The ratio of $\alpha, \beta$ will be increased from $1: 3$ to $3: 1$ while other parameters are the default values. Figure 8 shows the effect of the ratio of regulating factors on energy consumption when the sink location is updated for one time. The ratio is calculated by the number of node neighbors of $\alpha$ divided by the distance of the regulation cluster head to the center of the network in $\beta$. With the increasing of the ratio, the energy consumption of the network decreases rapidly and then continues to decrease slowly. When $\alpha: \beta=1: 1$, the network energy consumption reaches a minimum value which is about $0.015 \mathrm{~J}$. This is because when the ratio of $\alpha, \beta$ is smaller, the selection of cluster heads is depended on $f_{n}$. The best cluster head cannot be selected when the cluster heads are individually used and selected. Therefore the node chosen as the cluster head should have the maximum number of neighbor nodes and which are relatively closer to the center point.

\section{EVENT-INTENSIVE DATA COLLECTION PROTOCOL FOR WIRELESS SENSOR NETWORK}

The mobile sink can relieve the emergence of an "energy hole". However, if the network is divided into clusters unevenly, the clusters with a large scale will be invalid because of the heavy load. According to the above shortcomings and the scenes in which the events of interest frequently occur, a data collection protocol for wireless sensor networks with an approximately even division of clustering is put forward.

This protocol firstly analyzes the disadvantage of uneven distribution of the cluster heads and then it puts forward the principle of approximately even distribution of clusters. It is able to adapt to the frequent occurrence of events of interest with the two cases of direct communication of the cluster head and multiple hop communication of the cluster head being taken into consideration. When an event of interest occurs, the sink can take the initiative to move to the surrounding area for data collection.

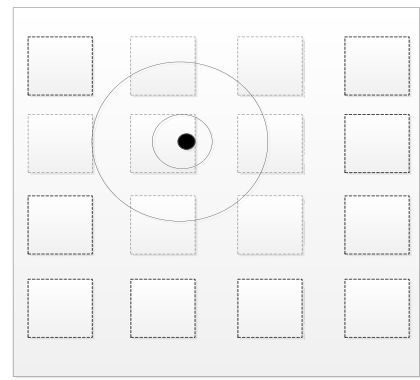

Figure 7. The influence of sink communication radius 


\section{A. ACBP protocol}

LEACH and other traditional methods for cluster division make the cluster head distribution uneven, and also may cause the load in each cluster to be unbalanced so as to form the energy holes early. In Figure 9, the black square indicates the cluster head and the dots represent the cluster members. The cluster heads A, B, C, D are not distributed evenly and they integrate in the lower area of the network. The number of the cluster members is respectively $21,8,5,10$. The more the members in the cluster is, the more data it should collect, so the load of cluster head A is larger than that of other cluster heads.

TABLE I.

W VALUE RELATIONS AND CANDIDATE NODES AVERAGE REMAINING ENERGY

\begin{tabular}{|c|c|}
\hline The candidate nodes average remaining energy & W \\
\hline 100 & 9 \\
\hline 96 & 8 \\
\hline 90 & 7 \\
\hline 85 & 6 \\
\hline 80 & 5 \\
\hline 75 & 4 \\
\hline 70 & 3 \\
\hline 65 & 2 \\
\hline
\end{tabular}

TABLE II.

PARAMETER SETTINGS

\begin{tabular}{|l|l|}
\hline \multicolumn{1}{|c|}{ Parameter } & \multicolumn{1}{c|}{ Value } \\
\hline Network side length & $900 \mathrm{~m}$ \\
\hline Communication radius & $30 \mathrm{~m}$ \\
\hline Node density & $0.001 / \mathrm{m} 2-0.005 / \mathrm{m} 2$ \\
\hline$e_{\text {elec }}$ & $50 \mathrm{~nJ} / \mathrm{bit}$ \\
\hline$\varepsilon_{f s}$ & $10 \mathrm{pJ} / \mathrm{bit} / \mathrm{m} 2$ \\
\hline$E_{d a}$ & $5 \mathrm{~nJ} / \mathrm{bitgsignal}$ \\
\hline$\alpha: \beta$ & $1: 1$ \\
\hline$h_{\text {neighbor }}$ & 6 \\
\hline
\end{tabular}

TABLE III

PARAMETER SETTINGS

\begin{tabular}{|l|l|}
\hline \multicolumn{1}{|c|}{ Parameter } & \multicolumn{1}{c|}{ Value } \\
\hline Network side length & $900 \mathrm{~m}-5400 \mathrm{~m}$ \\
\hline 1 & $180 \mathrm{~m}$ \\
\hline Communication radius & $30 \mathrm{~m}$ \\
\hline$e_{\text {elec }}$ & $50 \mathrm{~nJ} / \mathrm{bit}$ \\
\hline$\varepsilon_{f s}$ & $10 \mathrm{pJ} / \mathrm{bit} / \mathrm{m} 2$ \\
\hline$\varepsilon_{m p}$ & $0.0013 \mathrm{pJ} / \mathrm{bit} / \mathrm{m} 2$ \\
\hline$E_{d a}$ & $5 \mathrm{~nJ} / \mathrm{bitgsignal}$ \\
\hline$\alpha: \beta: \gamma: \delta$ & $1: 1: 1: 1$ \\
\hline$h_{\text {neighbor }}$ & 6 \\
\hline
\end{tabular}

TABLE IV.

PROTOCOL COMPARISON

\begin{tabular}{|c|c|c|c|c|}
\hline Protocol & $\begin{array}{c}\text { Sink } \\
\text { Number }\end{array}$ & $\begin{array}{c}\text { Network } \\
\text { structure }\end{array}$ & $\begin{array}{c}\text { Algorithm } \\
\text { type }\end{array}$ & $\begin{array}{c}\text { Application } \\
\text { type }\end{array}$ \\
\hline EDDGP & 1 & clustering & distributed & event-driven \\
\hline ACBP & 1 & clustering & distributed & event-driven \\
\hline
\end{tabular}

After data collection for a period of time, the remaining energy of $A$ is significantly smaller than other cluster heads. So the energy consumption is not balanced, which is not conducive to extending the survival time of the network. The number of the cluster members is respectively $13,9,11,11$ if the cluster heads are distributed evenly in A, B, C, D, which is show in Figure 9. As the number of their members and their energy consumption are similar to one another after a certain period of data collection, the even distribution of cluster heads can delay the emergence of an "energy hole", which is conducive to extending the survival time of the network.

In view of the above, a principle of approximately even distribution of clusters is proposed to avoid the low density of cluster heads and balance the load in the clusters.

When the interesting events occur frequently, a large number of data being transmitted to the sink will lead to a sharp increase in network load and the data channel around the sink will be crowded and blocked, resulting in a loss of data. What is more, once the sink position changes, the cluster head of information source which does not have time for updating will sent the data to the position where it stayed previously, which will cause a waste of energy. According to the situation, ACBP has to be improved. Once an event of interest occurs, the member nodes will send the information collected to the cluster heads which will then integrate to data and send a request packet for data collection to the sink. After receiving the request packet, the sink will quickly move to the cluster head for collecting information. Before moving, the sink will send the candidate region ID where the cluster heads of information source belongs to neighboring cluster heads with the maximum transmit power and then forward it to other clusters.

When the sink broadcasts the candidate region ID where the cluster heads of information source belong, the increasing of the transmission power can make more cluster heads directly receive the broadcast package. Figure 10 shows the situation when the sink improves the transmission power, where the triangle represents the sink, and the small circle represents the normal communication radius of the sink. At this time, only one cluster can communicate directly, and the remaining 15 clusters need to get information by using the multiple hop transmission. The large circle indicates the communication radius when the sink increases the transmission power, where now, it can communicate directly with the seven clusters and information only needs to be passed to the remaining nine clusters, which significantly reduces the range of transmission for multiple hop and reduces the energy consumption of nodes.

\section{B. Simulation experiment}

The experimental parameters are set as shown in Table 3. According to the conversion, $\mathrm{L}$ in the ACBP protocol corresponded in the adaptive region in the ALURP protocol as $180 \mathrm{~m}$.

Figure 10 shows the effect of $m_{1}, m_{2}$ on energy consumption of location updates of the sink. With the increasing of $m_{1}, m_{2}$, the energy consumption caused by the location update in the three protocols has raised significantly, and the energy consumption of the ALURP protocol is clearly higher than that of the $A C B P_{\text {multi-hop }}$ and $A C B P_{\text {l-hop. }}$. This is because every time a sink location is 
updated, the ALURP protocol needs to be flooded in an adaptive region or in the whole network while the two protocols of ACBP only need to notify the cluster heads to update the location of the sink. Because the number of nodes involved in Figure 10(a) is relatively small, so the total energy consumption is lower than that in Figure 10 (b). Additionally, the energy consumption of $A C B P_{\text {multi-hop }}$ is less than that of $A C B P_{\text {I-hop }}$, which also verifies the analysis in the previous section.

Figure 10 shows the effect of node density on the primary energy consumption of position update of the sink for one time. When the node density is equal, the energy consumption of position update of sink in the ALURP protocol is higher than that of $A C B P_{\text {multi-hop }}$ and $A C B P_{\text {l-hop }}$. With the increasing of density, the energy consumption of ALURP increases dramatically while the energy consumption of two protocols in ACBP increases slowly. As the node density is smaller in ACBP and the candidate nodes are fewer, which cannot fully exert the advantages of the criterion for approximately even clustering, so the difference in energy consumption between the two protocols of ACBP and ALURP is not significant; when the node density becomes larger, the candidate nodes are increased and the criterion of approximately even cluster distribution can select the best node as the cluster head, so the extent of increasing of energy consumption is much lower than that in the ALURP protocol.

Figure 10 shows the effect of the communication radius of the sink in $A C B P_{\text {multi-hop }}$ and $A C B P_{\text {I-hop }}$ protocols on energy consumption of the position update. When the communication radius of the sink is increased from $30 \mathrm{~m}$ to $180 \mathrm{~m}$, energy consumption when the sink location is updated for one time is gradually reduced. This is because with the increasing of the communication radius, the number of cluster heads directly receiving the sink broadcast packets increases with the amount of transfer data being reduced and the energy consumption of the network is reduced as well. Because the ways of communication between the two cluster heads are different, the decreasing speed of energy consumption is relatively slow which is slightly higher than that of $A C B P_{\text {multi-hop. }}$.

\section{COMPARISON OF PROTOCOLS}

Table 4 compares the four protocols proposed in this chapter. Both EDDGP and ACBP realize the data collection in the event-driven background by using the cluster distribution structure and one mobile sink. Furthermore, both protocols do not need to know the topology information of the whole network which is a distribution type of algorithm.

\section{SUMMARY}

This paper carries out research on two types of eventdriven data collection protocol of wireless sensor networks: EDDGP and ACBP protocols. In the EDDGP protocol, an adaptive evaluation model is put forward to select the cluster head, including the node classification, the fuzzy grade classification, the evaluation function and the parent grid resolution. Once an even of interest occurs, the member nodes will send the information collected to the cluster heads which will then integrate the data and send a request packet for data collection to the sink. After receiving the request packet, the sink will quickly move to

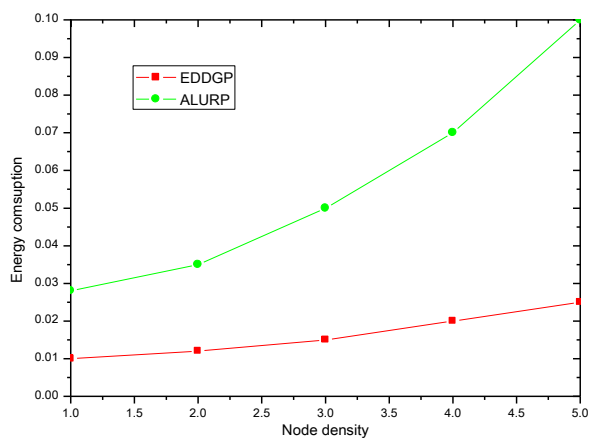

Figure 8. The influence of node density on energy consumption

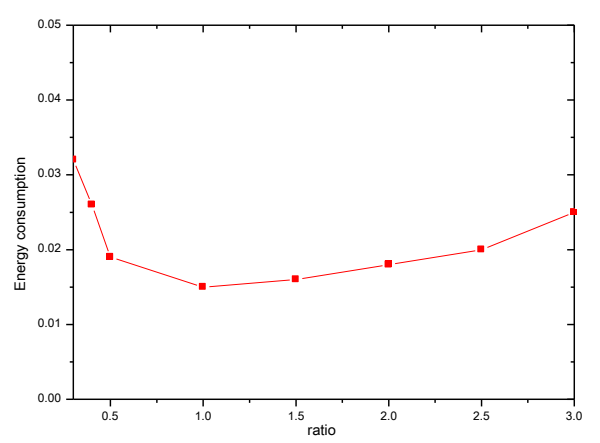

Figure 9. The influence of the ratio of energy consumption

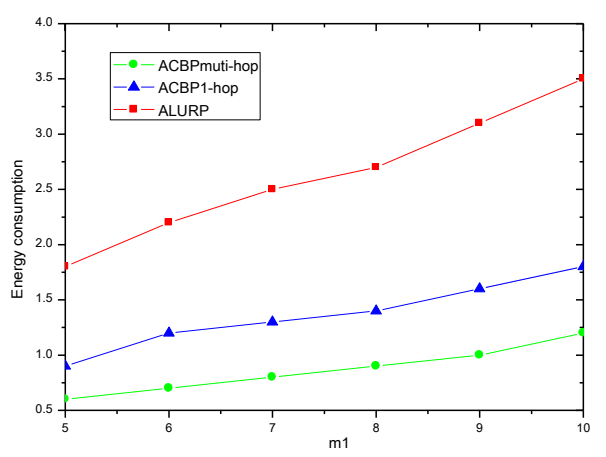

(a) $\mathrm{m} 1$

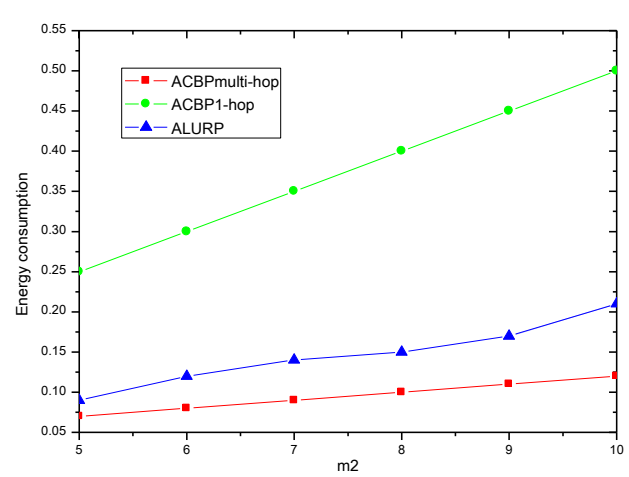

(b) $\mathrm{m} 2$

Figure 10. The energy consumption of $\mathrm{m} 1, \mathrm{~m} 2$ to the sink position update

the cluster head for collecting information. If the sink receives the request packet for data collection from other cluster heads of information sources in the moving process, it will save their grid ID. After collecting the data 
of the current cluster heads of the information source, the data of other cluster heads of information source are collected in turn from the nearest ones to the farthest ones. The theoretical research and simulation results show that EDDGP can reduce the energy consumption of location update of the sink so as to balance the energy consumption of nodes and prolong the lifetime of the network.

The ACBP protocol is put forward for situations where events of interest occur frequently. The protocol initially analyzes the disadvantage of uneven distribution of cluster heads and then it puts forward the criterion of approximately even cluster distribution with two circumstances of direct communication and multiple hop communication of cluster heads being taken into consideration as well. When the events of interest occur, the sink can take the initiative to move to the surrounding area of the events of interest for data collection. It carries out specific analysis from three aspects: the number times of movement for the sink, node density and communication radius.

\section{REFERENCES}

[1] C. Jongyoo, "Development and evaluation of an ambulatory stress monitor based on wearable sensors," IEEE Transactions on Information Technology in Biomedicine, Vol. 16, pp. 279-286, March 2012. http://dx.doi.org/10.1109/TITB.2011.2169804

[2] M. Kaushik, V. Ashok, "Robust regression using sparse learning for high dimensional parameter estimation problems," IEEE International Conference on Acoustics, Speech and Signal Processing - Proceedings, pp. 3846-3849, April 2010.

[3] C. Xingran, A. Maysam, "Brain death prediction based on ensembled artificial neural networks in neurosurgical intensive care unit," Journal of the Taiwan Institute of Chemical Engineers, Vol. 42, pp. 97-107, January 2011. http://dx.doi.org/10.1016/ j.jtice.2010.05.006

[4] W. Shuai, Z.Y. Sheng. "The simulation of mobile robots pursuit collision problem in wireless sensor network," Proceedings of the 27th Chinese Control Conference, pp. 190-193, April 2008.

[5] B. Julio, L. Carlos, "Using artificial intelligence in wireless sensor routing protocols," Lecture Notes in Computer Science, vol. 4251, pp. 475-482, June 2006. http://dx.doi.org/10.1007/11892960_58

[6] K. Jay, "A wireless sensor based solution that enables the enterprise to enable intelligent energy monitoring \& management using artificial intelligence," Technical Proceedings of the 2011 NSTI Nanotechnology Conference and Expo, vol. 3, pp. 799-800, June 2011.

[7] V. Constantin, "Environmental monitoring based on sensor networks and artificial intelligence," International Conference on Development, Energy, Environment, Economics - Proceedings, vol. 3, pp. 79-83, February 2010.

[8] J.Y. Li, J.P. Wang, "Wireless sensor network mobile agent routing based on the improved ant colony algorithm," Applied Mechanics and Materials, vol. 57, pp. 2339-2345, April 2014. http://dx.doi.org/10.4028/www.scientific.net/AMM.587-589.2339

[9] T.J. Lu, Y. Chen, "Application of improved ant colony algorithm technology in development of routing protocol in wireless sensor network," Applied Mechanics and Materials, vol. 32, pp. 583-586, March 2014. http://dx.doi.org/10.4028/www.scientific.net/amm.685.583

[10] F. Jiang, "Application of hybrid ant colony algorithm in wireless sensor network coverage," Computer Modelling and New Technologies, vol. 18(12), pp. 161-166, May 2014.

[11] Z. Sun, "Path optimization wireless sensor network based on ant colony algorithm," Journal of Chemical and Pharmaceutical Research, vol. 6, pp. 2086-2093, May 2010.

[12] J. Tian, M. Gao, "Wireless sensor network node optimal coverage based on improved genetic algorithm and binary ant colony algorithm," Eurasip Journal on Wireless Communications and Networking, vol. 1, pp. 236-239. June 2016. http://dx.doi.org/10.1186/s13638-016-0605-5

[13] X. Wu, L. Qi, "The optimization algorithm of wireless sensor network node based on improved ant colony," Sensors and Transducers, vol. 155, pp. 54-63. August 2013.

[14] R.F. Liu, "Fault diagnosis of wireless sensor based on ACO-RBF neural network," Proceedings-2010 3rd IEEE International Conference on Computer Science and Information Technology, ICCSIT 2010, pp. 248-251.

[15] T.Y. Li, "Fault diagnosis of auxiliaries in power plants based on wireless sensor networks with vibration transducer," Proceedings2010 2nd IEEE International Conference on Network Infrastructure and Digital Content, IC-NIDC 2010, pp. 732-736. http://dx.doi.org/10.1109/icnidc.2010.5657877

[16] B. Tang, B. Deng, L. Deng, "Mechanical fault diagnosis method based on multi-level fusion in wireless sensor networks," Zhendong Ceshi Yu Zhenduan/Journal of Vibration, Measurement and Diagnosis, vol. 36, pp. 92-96, April 2016.

[17] M.H. Lia, Z. Hua, S. Guang, "Energy aware routing algorithm for wireless sensor network based on ant colony principle," Journal of Convergence Information Technology, vol. 7, pp. 215-221, August 2012. http://dx.doi.org/10.4156/jcit.vol7.issue4.26

[18] Z. Sun, "Path optimization wireless sensor network based on ant colony algorithm," Journal of Chemical and Pharmaceutical Research, vol. 6, pp. 2086-2093, May 2010.

[19] J. Tian, M. Gao, "Wireless sensor network node optimal coverage based on improved genetic algorithm and binary ant colony algorithm," Eurasip Journal on Wireless Communications and Networking, vol. $1, \quad$ pp. 236-239. June 2016. http://dx.doi.org/10.1186/s13638-016-0605-5

[20] Y.C. Yao, Y. Yao, "The application of ant colony optimization in wireless sensor network routing," Advanced Materials Research, vol. 5, pp. 838-841. May 2013. http://dx.doi.org/10.4028/www. scientific.net/AMR.655-657.838

[21] J.Y. Zhang, D.Y. Chen, "Clustering routing algorithm ant colony optimization-based for wireless sensor network," Applied Mechanics and Materials, vol. 58, pp. 591-597, April 2015.

\section{AUTHORS}

Cunjiang $\mathbf{Y u}$ is with the Electronics and Information Engineering College, Changchun University, No.6543, Weixing Road, Changchun, Jilin Province, 130022, China (e-mail: 358219140@qq.com).

Submitted 03 September 2016. Published as resubmitted by the authors 25 October 2016. 